\title{
Téoros
}

Revue de recherche en tourisme

\section{Tourisme sportif}

Un essai de définition socio-économique

\section{Patrick Bouchet et Malek Bouhaouala}

Volume 28, numéro 2, 2009

Tourisme, sport et développement

URI : https://id.erudit.org/iderudit/1024801ar

DOI : https://doi.org/10.7202/1024801ar

Aller au sommaire du numéro

Éditeur(s)

Université du Québec à Montréal

ISSN

0712-8657 (imprimé)

1923-2705 (numérique)

Découvrir la revue

Citer ce document

Bouchet, P. \& Bouhaouala, M. (2009). Tourisme sportif : un essai de définition socio-économique. Téoros, 28(2), 3-8. https://doi.org/10.7202/1024801ar d'utilisation que vous pouvez consulter en ligne.

https://apropos.erudit.org/fr/usagers/politique-dutilisation/ 


\section{Tourisme sportif Un essai de définition socio-économique}

\author{
Patrick BOUCHET \\ Professeur des universités \\ Laboratoire Socio-Psychologie et Management du sport \\ Université de Bourgogne \\ patrick.bouchet@u-bourgogne.fr
}

\author{
Malek BOUHAOUALA \\ Maître de conférences \\ Université Joseph Fourier Grenoble 1 \\ malek.bouhaouala@ujf-grenoble.fr
}

Le tourisme sportif ne peut se prévaloir d'un héritage et d'une tradition dans l'histoire des vacances, du tourisme ou du sport. Il semble être un produit aux contours flous et aux limites en cours d'identification, caractérisé par la variété des métiers et des produits qui le constituent. En France, comme dans la plupart des pays occidentaux, l'intérêt porté au tourisme sportif est né d'un double mouvement. L'un décrit l'association et l'intégration croissante de pratiques et représentations traditionnellement véhiculées par le sport en tant que loisir dans les produits touristiques. L'autre caractérise l'amplification sociale des loisirs sportifs qui se fondent de plus en plus dans les pratiques sociétales, échappant ainsi au cadre spatiotemporel de l'univers sportif. En récusant l'idée de faire du tourisme un concept «cannibale» qui se déclinerait en catégories en fonction des orientations choisies, le tourisme sportif semble relever d'un processus original de métissage dont les repères évoluent.

Fondé à la fois sur l'évolution du sport classique vers les loisirs sportifs et sur la massification et la diversification du tourisme, le tourisme sportif revêt un intérêt croissant suscité par l'impact économique qui lui est associé et l'attractivité qu'il génère en raison des valeurs et des représentations qu'il véhicule (environnement, écologie, liberté, souci du bien être, etc.). Ces éléments porteurs font que la communication institutionnelle du tourisme se réalise de plus en plus avec le support des activités sportives (Pigeassou, 2002). En outre, sa dynamique provoque des débats qui sont au cœur de la réflexion actuelle concernant le développement territorial, le commerce équitable et la gestion des ressources. Par ailleurs, le tourisme sportif ne constitue pas un secteur uniformément identifié dans tous les pays du monde comme cela peut l'être en Amérique du Nord, en Australie ou au Mexique, par exemple. Pour qui veut s'aventurer à connaître le tourisme sportif dans la panoplie d'informations disponibles au niveau français ou au niveau international, le constat est double. D’une part, les données accessibles sont limitées et, hormis des revues comme The Journal of Sport and Tourism ou les Cahiers Espaces, proviennent généralement d'études commanditées par des agences spécialisées ou des organisations (relevant de la puissance publique) qui publient des statistiques sur le tourisme en fonction de leur spécificité ou pour nourrir leurs propres projets. D'autre part, l'identification du tourisme sportif comme vecteur du tourisme est embryonnaire et très souvent limitée à une identification des «sports structurants du tourisme» (Origet de Cluzeau et Vicérat, 2000; Kurtzman et Zauhar, 2003).

\section{Des réflexions récentes sur l'objet " tourisme sportif » au niveau international}

Des travaux précurseurs de chercheurs s'interrogeaient sur ce qui est caractéristique du tourisme sportif. Le fait de participer aux grands évènements sportifs (Jeux olympiques, Championnats du monde, Coupe du monde...) associe des prestations touristiques à des services sportifs même si le consommateur est passif (Hall, 1992; Chalip, Green et Vander, 1998; Delpy, 1996; Gibson, 1998). La participation à des pratiques sportives ou à des loisirs sportifs sur un lieu de vacances constitue une autre catégorie d'observations combinant sport et tourisme (De Knop, 1987, 1990 ; Gibson et Yiannackis, 1992, 1994; Nogawa, Yamguchi et Hagi, 1996). D’autres réflexions portent sur la visite de lieux culturels en rapport avec le sport (Redmont, 1990, 1991; Gibson, Attle et Yiannakis, 1998) : musées, monuments, installations sportives, attractions. Entre ces catégories d'observations, il existe un élément commun : l'association ou la combinaison du terme «sport», ou du moins d'éléments du sport, avec des services touristiques.

Par ailleurs, la réflexion des chercheurs sur le tourisme sportif a donné lieu à des propositions ou à des contributions pour le définir. Plus ou moins techniciennes ou catégorielles, ces définitions ont eu le mérite d'ouvrir le chantier, mais elles provoquent deux commentaires. Premièrement, elles ont pour point de départ une approche fondée sur la répétition 
d'observations de pratique. Deuxièmement, les faits observés sont fondés sur des catégories distinctes : les usages, les produits ou la nature de l'expérience. On retiendra de ces travaux la prééminence de l'implication sportive sous l'angle de l'investissement et sous celui du type d'expérience. Ce qui est en jeu dans ce souci de l'implication, c'est la difficulté à considérer que peuvent être qualifiées de sportives des activités non directement et essentiellement motrices, mais relevant de l'univers culturel du sport, qui est lui-même lié à des définitions restrictives et, encore une fois, techniciennes du sport.

La prise de conscience du sport comme un vecteur des produits touristiques a fait son chemin sans toutefois s'inscrire dans une réflexion systématique articulée sur la compréhension de la valeur ajoutée du tourisme sportif et sur l'explication de son organisation. Les contributions citées et qui sont apparues des plus significatives dans la littérature sur ce sujet sont présentées dans le tableau 1 issu des travaux de Pigeassou (2004).

\section{Le développement socio-économique du tourisme et du sport dans le monde}

Le tourisme international a connu un développement spectaculaire depuis la fin de la Seconde Guerre mondiale, même si son rythme de croissance s'est ralenti depuis 1980. En effet, les arrivées touristiques à l'échelle mondiale ont été multipliées par 10 entre 1960 (69,3 millions) et 2000 (697,6 millions) (Direction du tourisme, 2008). En 2008, selon l'Organisation de coopération et de développement économique (OCDE), le tourisme était un des moteurs de la mondialisation et représentait pour les pays membres un des secteurs les plus dynamiques. En effet, ces derniers représentaient $70 \%$ de l'activité touristique mondiale et le secteur constituait $30 \%$ de leur exportation de services. La même année, selon le World Travel and Tourism Council (WTTC), le tourisme représentait 9,9\% du PIB mondial ( $10 \%$ en 2002), $11 \%$ des exportations (11,9\% en 2002) et $8,4 \%$ de l'emploi mondial. Au sein de l'Union européenne, le tourisme contribue actuellement à $4 \%$ du PIB,

\begin{tabular}{|c|c|}
\hline \multicolumn{2}{|r|}{ Tableau 1: Les contributions à la définition du tourisme sportif } \\
\hline \multicolumn{2}{|r|}{ Définitions à partir de l'observation des usages } \\
\hline De Knop (1987) & Une personne qui, en vacances, pratique une activité sportive \\
\hline Redmond $(1990,1991)$ & $\begin{array}{l}3 \text { types d'usage : } \\
\text { - spectateur d'évènements sportifs; } \\
\text { - participation active dans l'activité sportive; } \\
\text { - visiteur d'éléments de la culture sportive (musées, monuments, installations, célébrations de la mémoire du sport). }\end{array}$ \\
\hline De Knop (1990) & $\begin{array}{l}3 \text { types de participation sportive pendant les vacances: } \\
\text { - participer à une activité sportive ou ludosportive (the pure sport holiday); } \\
\text { - participer à des activités sportives ou ludosportives en utilisant les possibilités offertes (installations, services) } \\
\text { sur le lieu de vacances; } \\
\text { - participer occasionnellement à des activités sportives ou ludosportives non organisées sur le lieu de vacances. }\end{array}$ \\
\hline Hall (1992) & $\begin{array}{l}2 \text { types d'usage : } \\
\text { - déplacement pour regarder; } \\
\text { - déplacement pour participer dans un cadre sportif ou ludosportif. }\end{array}$ \\
\hline Nogawa et al. (1996) & $\begin{array}{l}3 \text { types d'usage : } \\
\text { - voyager pour prendre part à un évènement sportif; } \\
\text { - voyager pour assister à un spectacle sportif; } \\
\text { - voyager pour participer à des activités sportives auto-organisées. }\end{array}$ \\
\hline \multicolumn{2}{|c|}{ Définitions à partir de l'observation des productions caractéristiques du tourisme sportif } \\
\hline $\begin{array}{l}\text { Kurtzman (1996) } \\
\text { Kurtzman et Zauhar (2003) }\end{array}$ & $\begin{array}{l}5 \text { catégories de produits principaux : } \\
\text { - sport tourism attraction : le tourisme sportif ciblé sur l'utilisation des attractions sportives (musées sportifs, congrès, con- } \\
\text { férences sportives, exhibitions et démonstrations sportives, parcs sportifs [aquatiques en particulier], zones de descente en } \\
\text { radeau, golfs, pistes et aménagements pour le ski, stades, patinoires, etc.); } \\
\text { - sport tourism ressorts : les séjours de tourisme sportif dans des centres, des stations ou des camps de loisir ou } \\
\text { d'entraînement sportif; } \\
\text { - sport tourism cruises : les croisières à objectif sportif dont l'objet est un sport, la rencontre de sportifs, les visites de lieux } \\
\text { sportifs, etc.; } \\
\text { - sport tourism tours : les voyages de tourisme sportif pour l'exercice de la pratique d'une activité sportive : golf, tennis, } \\
\text { randonnée, safari, trekking, etc.; } \\
\text { - sport tourism events : le tourisme sportif à l'occasion d'un évènement régional, national ou international (rencontre interna- } \\
\text { tionale, Jeux olympiques...). }\end{array}$ \\
\hline \multicolumn{2}{|r|}{ Définition à partir du type d'expérience } \\
\hline Standeven et De Knop (1999) & $\begin{array}{l}\text { Le tourisme sportif comme expérience culturelle à deux dimensions : } \\
\text { - l'activité physique; } \\
\text { - le lieu de réalisation. }\end{array}$ \\
\hline
\end{tabular}


(ce pourcentage variant entre environ $2 \%$ et $12 \%$ selon les états membres); sa contribution indirecte à la formation du PIB est bien supérieure puisque le tourisme génère indirectement plus de $10 \%$ du PIB européen et représente plus de $12 \%$ de l'emploi total (Direction du tourisme, 2008). Néanmoins, dans leur rapport de 1982, les experts de l'Organisation mondiale du tourisme (OMT) ont défini que «le rôle économique du tourisme ne se limite pas à ses effets au niveau international. C'est au niveau national que se manifestent, avec la plus grande intensité, ses effets étant donné que le tourisme peut être, en tant que secteur productif, un facteur de développement et de redistribution de l'activité économique». Ainsi, le tourisme en tant que secteur socio-économique identifié joue un rôle fondamental dans l'économie française. Il a généré en 2007 une consommation de l'ordre de 117,6 milliards d'euros et 894000 emplois salariés (Direction du tourisme, 2008).

Dans le même temps, les motivations des vacanciers sont devenues à la fois simples en termes d'aspirations (repos, dépaysement, convivialité familiale ou amicale) et complexes en termes de disponibilité et de pouvoir d'achat (fractionnement des vacances, frontières de plus en plus floues entre travail et loisirs, etc.). Un autre paradoxe concerne l'augmentation des courts séjours permettant une rupture avec le rythme du quotidien, avec des aspirations à des séjours plus longs afin de prendre le temps de vivre (Touriscopie, 2003). Ces touristes oscillent aussi entre achats de dernière minute, selon les envies et les disponibilités, et achats planifiés longtemps à l'avance. Cependant, face à la standardisation et à l'uniformisation de l'offre (villages de vacances, centres urbains, commerces, parcs de loisirs, etc.), certains vacanciers souhaitent devenir de «nouveaux voyageurs » qui privilégient l'émotion, les rencontres culturelles et humaines. D'autres sont aussi à la recherche d'authenticité (vivre les traditions et les cultures du pays), d'enchantement (donner du sens, vivre des émotions, découvrir l'autre). Cette quête d'authenticité est parfois difficile à combler lorsque les traditions se perdent. Pour y remédier, certains acteurs (pays, villes, etc.) n'hésitent pas à thématiser certains parcs (Puy du Fou en Vendée, Dracula en Roumanie, etc.), certains villages (faux villages mexicains), etc. (Touriscopie, 2003) «Le tourisme aujourd'hui est plus une mise en scène commerciale qu'une expérience culturelle. Quand l'ambiance locale ne fournit pas son lot d'expériences, on se charge de le recréer artificiellement pour que chaque client ait droit à son lot de poncifs exotiques» (Rifkin, 2002). Cette tendance se retrouve dans des parcs de type «Disneyland Paris» ou des stations touristiques de type «Arc $1950 »$. Les envies des touristes sont partagées entre repos et activités, et ce, au sein d'un même séjour. Ils ont des pratiques très diversifiées pendant leurs vacances. En effet, si $30 \%$ des personnes ne pratiquent pas d'activité particulière, $30 \%$ choisissent des activités culturelles et $54 \%$ pratiquent une activité physique durant leur séjour en France et à l'étranger (Direction du tourisme, 2008).

Le sport, d'une manière globale, représente environ $2 \%$ du PIB dans les pays riches de la planète. Rien que la consommation mondiale d'articles de sport a atteint 182 milliards d'euros pour la seule année 2006 (NPD Market Research Group). En Amérique du Nord, selon une étude du Convention Bureau's
North American le sport compte pour $25 \%$ et $34 \%$ respectivement des recettes du tourisme et de l'évènementiel (Kurtzman and Zauhar, 2003). En France, les dépenses liées au sport ont atteint 31,7 milliards d'euros pour l'année 2006. Les ménages qui pratiquent un sport régulièrement représentent entre $50 \%$ et $85 \%$ de la population selon les différentes estimations. Leurs dépenses sportives forment la moitié des dépenses globales aux alentours de 15,7 milliards d'euros pour l'année 2005 selon l'Institut national de la statistique et des études économiques. Ce qui fait des ménages les premiers à financer l'industrie du sport. Toutefois, les Français ne sont pas considérés comme de gros consommateurs de produits sportifs en Europe, car ils ont, en outre, la réputation de préférer les pratiques "sauvages» aux pratiques réglementées et de bouder les équipements publics comme les stades et les piscines (Halba, 1997). La consommation de biens et services sportifs représente 1,6\% de la consommation finale des ménages depuis une dizaine d'années. Toutefois, la part des services est passée de $36 \%$ en 1995 à $43 \%$ en 2006 et, actuellement, les achats de services sportifs par les ménages représentent 6,8 milliards d'euros pour l'année 2006 (Ministère de la Santé, de la Jeunesse et des Sports [MSJS], 2008). C'est le poste de dépense pour lequel la consommation des ménages a le plus augmenté en France. La consommation de services sportifs s'étant fortement développée et transformée, l'offre a dû s'adapter. En effet, il apparaît que l'usager d'un service public municipal ou l'adhérent d'un club sportif, sont aussi des consommateurs qui vont exiger de l'offre non marchande la qualité de service à laquelle ils sont habitués dans le secteur commercial, et davantage parfois (l'éthique en sus).

Les motivations des sportifs français étant, pour 9 pratiquants sur 10, la santé, la détente et le plaisir (plaisir du sport et plaisir de se retrouver entre amis ou en famille), les prestataires sont en face d'une demande de sports de loisirs difficile à cerner (MSJS, 2008). En effet, bon nombre d'autres loisirs non sportifs permettent aussi de satisfaire de tels besoins (on peut se détendre et cultiver sa forme en se promenant à la campagne). En effet, le spectre des pratiques étant très large, il va capter une partie non négligeable de cette demande indécise quant à la meilleure utilisation d'un temps libre grandissant. Il s'agit d'un segment qui intéresse tout particulièrement l'offre marchande, notamment certains sous-segments comme celui des seniors particulièrement motivés et disposant d'un bon pouvoir d'achat.

\section{Le poids du tourisme et du sport dans le développement social et économique}

En somme, le poids socio-économique des marchés du tourisme et du sport est significatif d'un rôle social et économique fondamental. Par conséquent, il est possible d'avancer l'idée que le secteur du tourisme sportif (issu du croisement des deux), même si l'on ne dispose pas encore de données précises le concernant, occupe une place importante dans le développement socio-économique. Cette importance est aussi mise en avant dans les politiques de développement des territoires et des états. Le tourisme sportif trouve ainsi sa place dans les dimensions fondamentales de l'organisation sociale que ce soit à l'échelle nationale ou mondiale : 
- au niveau politique, il sert de levier de développement, de cadre d'aménagement des territoires, de guide pour la préservation et la gestion des ressources naturelles, etc.;

- au niveau social, au-delà du lien qu'il crée entre les individus, il joue un rôle dans l'intégration des populations par l'emploi, il participe à l'éducation à la citoyenneté et à l'environnement pour les autochtones et les allochtones, etc.; - au niveau économique, c'est un véritable secteur entrepreneurial, plus ou moins saisonnier selon les territoires et les activités, constituant un moteur de la création d'entreprises, d'emplois, d'activités de services, etc.

$\mathrm{Au}$ regard de sa complexité et de son importance, le tourisme sportif se trouve être récupéré, voire instrumentalisé, par différents acteurs : entreprises, états, fédérations sportives, consommateurs... lesquels y investissent et s'y investissent. Les enjeux, les objectifs et les logiques de ces nombreux acteurs sont à ce point variés et multiples qu'ils constituent une organisation complexe et large en même temps. Certes, le tourisme sportif est devenu une véritable source de revenus, d'activités et d'emplois pour les territoires touristiques, mais il l'est également pour les espaces supports (territoires pourvoyeurs de pratiquants ou lieux d'accueil et/ou de transit).

Ceci étant, bien que les chiffres clés concernant le tourisme sportif ne soient pas connus, en croisant ceux des marchés du tourisme et du sport, on peut faire l'hypothèse qu'ils sont également importants. Cependant, il est possible de fournir quelques chiffres indicatifs concernant certains segments du secteur du tourisme sportif ou certaines activités localisées. Par exemple, les territoires touristiques "campagne et montagne", fortement liés aux loisirs sportifs, représentent respectivement $28,2 \%$ et $7,5 \%$ de la consommation touristique française. Ainsi, l'industrie des sports d'hiver génère une richesse assez importante : pour la saison 2008-2009, les remontées mécaniques ont réalisé un chiffre d'affaires $(\mathrm{CA})^{1}$ estimé à plus de 1,1 milliard d'euros (Protourisme, 2009). Autre exemple encore, le tourisme équestre a réalisé un CA estimé à plus de 300 millions d'euros pour 2004 (Bouhaouala et Albertini, 2005; données révisées lors d'une seconde enquête en 2006). Il convient alors de reconnaître le potentiel qu'offrent, d'une part, les loisirs sportifs au développement social et économique des territoires touristiques et, d'autre part, le tourisme à la diversification des loisirs sportifs. Par le biais du tourisme sportif, le sport et le tourisme, d'une manière générale, sont fortement liés in fine à la consommation dans les territoires touristiques et à la dynamique de développement socio-économique. Ainsi, ce secteur n'est pas un simple partenariat à sens unique entre le sport et le tourisme qui sert à mieux comprendre comment le dernier utilise le premier pour se renforcer économiquement comme le prétendent Kurtzman et Zauhar (2003). Il s'agit d'un nouveau sous-secteur économique dans lequel il convient plutôt de parler d'une interaction du sport et des loisirs qui génère un nouveau modèle économique.

Si l'importance sociale, politique et économique du tourisme sportif au plan national et mondial n'est plus à démontrer dans le mode de vie des pays occidentaux et riches, il représente également aujourd'hui pour les pays émergents et en voie de développement un véritable enjeu de progrès social et économique, voire une véritable aubaine en termes de rentrée de devises pour tenter une reconversion réussie de leurs systèmes socio-économiques. De ce fait, il devient alors justifié de ne pas s'attarder sur les aspects techniques ou catégoriels du tourisme sportif pour se poser les questions nécessaires à la compréhension de son organisation générale, et pour mesurer la place de ce dernier dans le développement social et économique. Ceci à partir de choix, d'une part, d'approches pluridisciplinaires (théoriques et méthodologiques) et, d'autre part, de thématiques exemplaires. En conséquence, il faut comprendre dans la suite du texte que toute référence au terme «tourisme sportif » renvoie aux loisirs sportifs consommés en relation avec le secteur ou les territoires touristiques même en l'absence de nuitée. Dans ce contexte de massification et de diversification des marchés du tourisme et du sport, il devient maintenant nécessaire de définir le tourisme sportif et de circonscrire son marché.

\section{Essai de définition socio-économique du tourisme sportif}

Loin d'être imperméables, les marchés du tourisme et du sport se sont suffisamment imbriqués pour constituer aujourd'hui une véritable source de revenus et d'emplois pour, d'une part, les territoires et les régions touristiques et, d'autre part, les pays émetteurs et récepteurs. Bien plus qu'un simple argument de vente, comme pourrait l'être l'évènementiel sportif, les loisirs sportifs forment désormais un élément constitutif à part entière du marché des vacances. Alors, circonscrire ou réduire l'association "tourisme et sport» aux seuls treks extrêmes (dans la jungle ou l'Himalaya) ou aux traversées transatlantiques est loin de refléter son étendue et sa réalité. L'extension observée dans le tourisme sportif tant du côté de la demande que de l'offre incite les chercheurs de multiples champs scientifiques (géographie, sociologie, économie, gestion, etc.) à s'interroger sur la structure et la portée de ce nouveau sous-secteur socio-économique au moment même où ses principaux acteurs tentent de relever les défis que pose le développement durable des territoires et le commerce à l'échelle de la mondialisation.

Fort de ce constat, notre essai de définition du tourisme sportif part des enjeux socio-économiques liés au triptyque «tourisme-sport-développement», le but n'étant pas de produire une définition restrictive et technicienne, mais de poser les questions empiriques fondamentales liées à la compréhension de l'organisation et au développement de ce secteur tant à l'échelle locale que globale. Il s'agit également de poser des regards croisés issus de plusieurs disciplines et diverses approches. En outre, cette définition doit aussi être l'occasion d'identifier les différents enjeux, présents et à venir, relatifs au tourisme sportif aussi bien pour les territoires, les entreprises, les collectivités publiques que pour les consommateurs. De ce fait, notre tentative de définition s'appuie sur une vision inductive et empirique du tourisme sportif en partant de sa réalité et non de ce qu'il devrait être du point de vue du théoricien. Autrement dit, il est plus heuristique de définir le tourisme sportif à partir de la place qu'il occupe sur le plan socio-économique et de ses axes de développement. Il est plus constructif d'aborder le tourisme sportif comme une activité générant de la valeur sociale et 
économique afin de saisir tous ses aspects et ses impacts. En effet, lorsque des individus ou des groupes d'individus achètent des loisirs sportifs dans un territoire touristique, ils génèrent de l'activité sociale et économique même si cela ne nécessite pas un déplacement avec nuitée.

Cette vision ouverte permet donc de prendre en compte la complexité de la filière et de porter un intérêt autant aux activités la composant qu'à l'organisation des prestataires et aux modes de dépenses générés. Ainsi, la seule comptabilisation $\mathrm{du}$ nombre de voyageurs et des nuitées n'est plus suffisante pour estimer le rôle véritable du tourisme en général et du tourisme sportif en particulier. Pourtant, la prise en compte des chiffres d'affaires du secteur et de ses composantes professionnelles permet une vision conforme à la réalité. La seule définition fondée sur le déplacement avec nuitée hors du domicile, bien qu'elle soit pratique, est insuffisante pour définir le tourisme sportif et saisir son importance du point de vue socio-économique. Par conséquent, si l'on souhaite appréhender le tourisme sportif comme une activité générant de la valeur socio-économique, cela nécessite une vision inductive et croisée, centrée sur ses axes de développement, ses impacts et ses modes de diversification avec d'autres soussecteurs touristiques ou de loisirs.

C'est dans cet esprit que ce numéro spécial de la revue Téoros rassemble sept contributions articulées autour de trois grandes thématiques. La première est focalisée sur la problématique liée au développement des territoires par le biais du tourisme sportif, que ce soit à travers le prisme du kayak (De Myttenaere et D'Ieteren), de l'eau vive australe (Marsac) ou des stations balnéaires (Bodin et al.). Ces trois premières contributions démontrent toutes la complexité inhérente à l'essor des activités de plein air au regard des préoccupations qu'éprouvent les différents acteurs.

La deuxième thématique s'intéresse davantage à la problématique de l'impact des activités et à la mesure des réelles retombées du tourisme sportif. L'étude des événements comme les marathons (Lapeyronie) ou une Coupe du monde de rugby (Charrier) montre la complexité et la difficulté méthodologique que pose l'évaluation des impacts locaux et nationaux de ce type de manifestation.

La troisième et dernière thématique se focalise plus particulièrement sur la diversification du tourisme sportif vers de nouveaux territoires comme le surf en ville (Lefebvre) ou de nouveaux services «nature-culture-sport» (Guyon). Ces deux dernières contributions démontrent, si besoin était, que ce sous-secteur est en perpétuelle transformation, innovant au gré des actions engagées par les prestataires pour attirer et satisfaire des clients dont les profils et les envies évoluent sans cesse.

Note

1 CA : le chiffre d'affaires est une représentation financière du volume de l'activité économique d'un secteur ou d'une entreprise, il ne s'agit donc pas de la plus-value dégagée. Là réside donc la différence avec le PIB.

\section{Bibliographie}

BOUHAOUALA, Malek et François ALBERTINI (2005) «La nécessaire professionnalisation du tourisme équestre : structure financière et commerciale des centres équestres ", Espaces tourisme et loisirs, n 229 , septembre, p. 16-18.

CHALIP, Laurence; Christine GREEN et Velden VANDER (1998) «Sources of Interest in Travel to the Olympic Games ", Journal of Vacation Marketing, ${ }^{\circ}$ 4, p. 7-22.

De KNOP, Paul (1990) «Sport for all and active tourism», World Leisure and Recreating, $\mathrm{n}^{\circ} 32$, p. 30-36.

De KNOP, Paul (1987) «Some Thoughts on the Influence of Sport Tourism ", dans Netenya, Proceedings of the international seminar and workshop on outdoor education, recreation and sport tourism, Wingate Institute for Physical Education and Sport, p. 38-45.

DELPY, Lisa (1996) «A Comparaison of the Pre and Post Economic Impact Figures Reported for the 1994 World Cup ", Communication présentée à la $11^{e}$ conférence annuelle de la Société Nord Américaine de management du sport, Université de New Brunswick, Moncton.

Direction du tourisme (2008) Mémento du tourisme, Rapport.

Direction du tourisme (2008) Tourisme des français : les activités des touristes lors de leurs voyages, Bulletin statistiques et d'études, Tourisme infos stat $n^{\circ} 2008-7$.

Direction du tourisme (2005) Chiffres clés du tourisme, Bulletin statistiques et d'études, $8 \mathrm{p}$.

GIBSON, Helen Jamieson (1998) «Sport Tourism: a Critical Analysis of Research », Sport Management Review, n 1, p. 45-76.

GIBSON, Helen Jamieson; Simon ATTLE et Andrew YIANNAKIS (1998) "Segmentating the Sport Tourist Market: a Lifespan Perspective», Journal of Vacation Marketing, $\mathrm{n}^{\circ}$ 4, p. 52-64.

GIBSON, Helen Jamieson et Andrew YIANNAKIS (1994) «Some Characteristics of Sports Tourists: a life Spain Perspective», Communication présentée à la conférence annuelle pour la Société Nord Américaine de sociologie du sport, Savannah.

GIBSON, Helen Jamieson et Andrew YIANNAKIS (1992) «Some Correlates of the Sport Lover (Tourist): a Life Course Perspective», Communication présentée à la Société Nord Américaine de sociologie du sport, Toledo.

HALBA, Bénédicte (1997) Économie du sport, Paris, Économica, 112 p.

HALL, C. Michael (1992) Hallmark Tourist Events: Impacts, Management and Planning, Londres, Belhaven Press, 215 p.

INSEE (2005) «Pratique sportive et activités culturelles vont souvent de pair», Bulletin statistiques et d'études, INSEE première, $n^{\circ} 1008$, mars.

KURTZMAN, Joseph et John ZAUHAR (2003) «A Wave in Time - The Sports Tourism Phenomena ", Journal of Sport and Tourism, $\mathrm{n}^{\circ}$ 8:1, p. 35-47.

Ministère de la Santé de la Jeunesse et des Sports (MSJS) (2008) «Les chiffres clés du sport », Bulletin statistiques et d'études.

NOGAWA, Haruo; Yasuo YAMGUCHI et Yumiko HAGI (1996) «An Empirical Research Study on Japanese Sport Tourism in Sport-for-All Events: Case Studies of a Single-Night Event and a Multiple-Night Event », Journal of Travel Research, $\mathrm{n}^{\circ} 35, \mathrm{p}$. 46-54.

NPD Market Research Group (2006) <http://www.npd.com/ corpServlet?nextpage=corp_welcome.html>, consulté le 24 août 2009.

Organisation de coopération et de développement économique (OCDE) $(2008)<$ http://www.oecd.org/home/0,3305,fr_2649_201185_1_1_1_1 _1,00.html/>, consulté le 24 août 2009.

Organisation mondiale du tourisme (OMT) (1982) <http://www.unwto. org/index_f.php/>, consulté le 24 août 2009.

ORIGET DE CLUZEAU, Claude et Patrick VICÉRAT (2000) «Le tourisme des années 2010 : La mise en future de l'offre», Paris, La Documentation Française, 244 p. 
PIGEASSOU, Charles (2004) «Le tourisme sportif : une réalité sociale aux contours incertains", Le tourisme sportif, Sobry (dir), Villeneuve d'Asq, Presse Universitaire du Septentrion, $384 \mathrm{p}$.

PIGEASSOU, Charles (2002) «Sport Tourism, a Growing Sector: Issues and Perspectives in France », dans Sean Gammon et Joseph Kurtzman (éd.), «Sport tourism: principles and practice, Eastbourne», Leisure Studies Association, p. 129-140.

Protourisme (2009) Communiqué de Presse, 22 avril.

REDMOND, Gerald (1991) «Changing Styles of Sports Tourism: Industry/ Consumer Interactions in Canada, the USA and Europe», dans M. T. Sinclair et M. J. Stabler (éd.), The Tourism industry: an international analysis, Wallingford, CAB International, p. 107-120.
REDMOND, Gerald (1990) «Points of Increasing Contact: Sport and Tourism in the Modern World", dans A. Tomlinson (éd.), Proceedings of the Leisure Studies Association, Second international conference, Leisure, Labour, and Lifestyles: international Comparisons, Eastbourne, LSA Publication, p. 158-169.

RIFKIN, Jeremy (2002) L'âge de l'accès, Paris, Pocket, 383 p.

STANDEVEN, Joy et Paul De KNOP (1999) Sport tourism, Champaign,

Human Kinetics, 1999.

Touriscopie (2003), $\mathrm{n}^{\circ}$ 40, mars-avril, $22 \mathrm{p}$.

World Travel and Tourism Council (WTTC) (2008) <http://www.wttc.org/>, consulté le 24 août 2009.

\section{www.teoros.uqam.ca Un outil pour vos recheches}

\section{Profil}

Éditions : courante et future Index des articles

Abonnements

Appel à textes

Colloques

Ressources et liens

Coordonnées

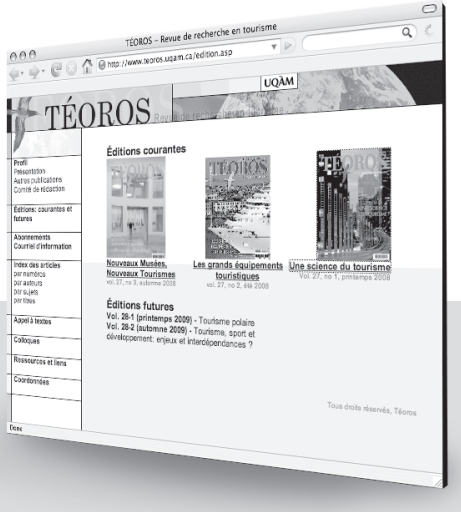

Venez découvrir le résumé indexé de plus de 400 articles portant sur le tourisme!

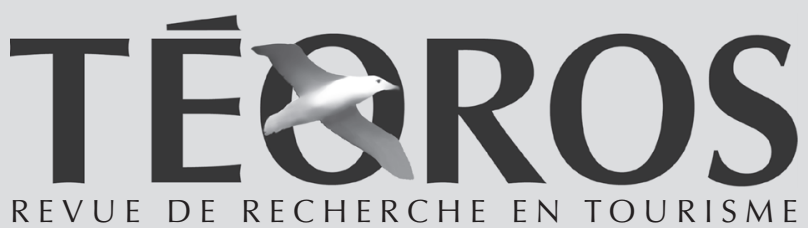

